\title{
Genetic Analysis of Age-at-Onset for Cardiovascular Risk Factors in a Brazilian Family Study
}

\author{
Suely Ruiz Giolo ${ }^{a, c} \quad$ Alexandre Costa Pereira $^{a} \quad$ Mariza de Andrade $^{d}$ \\ Camila Maciel de Oliveira ${ }^{a} \quad$ José Eduardo Krieger ${ }^{a} \quad$ Júlia Maria Pavan Soler ${ }^{b}$ \\ ${ }^{a}$ Genetics and Molecular Cardiology Laboratory, Heart Institute, and ${ }^{b}$ Department of Statistics, University of \\ Sao Paulo, Sao Paulo, ' Department of Statistics, Federal University of Parana, Parana, Brazil; ${ }^{\text {d Department of }}$ \\ Health Sciences Research, Mayo Clinic, Rochester, Minn., USA
}

\section{Key Words}

Censored trait · Complex disease $\cdot$ Genetic models •

Survival analysis

\begin{abstract}
Background/Aims: Statistical analysis of age-at-onset involving family data is particularly complicated because there is a correlation pattern that needs to be modeled and also because there are measurements that are censored. In this paper, our main purpose was to evaluate the effect of genetic and shared family environmental factors on age-at-onset of three cardiovascular risk factors: hypertension, diabetes and high cholesterol. Methods: The mixed-effects Cox model proposed by Pankratz et al. [2005] was used to analyze the data from 81 families, involving 1,675 individuals from the village of Baependi, in the state of Minas Gerais, Brazil. Results: The analyses performed showed that the polygenic effect plays a greater role than the shared family environmental effect in explaining the variability of the ageat-onset of hypertension, diabetes and high cholesterol. The model which simultaneously evaluated both effects indicated that there are individuals which may have risk of hypertension due to polygenic effects $130 \%$ higher than the overall average risk for the entire sample. For diabetes and high cholesterol the risks of some individuals were 115 and 45\%,
\end{abstract}

respectively, higher than the overall average risk for the entire population. Conclusions: Results showed evidence of significant polygenic effects indicating that age-at-onset is a useful trait for gene mapping of the common complex diseases analyzed. In addition, we found that the polygenic random component might absorb the effects of some covariates usually considered in the risk evaluation, such as gender, age and BMI.

Copyright $\odot 2009$ S. Karger AG, Basel

\section{Introduction}

Genetic complex diseases that exhibit variation in ageat-onset have in recent years motivated the development of more general methodologies in order to investigate the role of genetic and environmental effects on age-at-onset. Statistical analysis of age-at-onset involving family data is particularly complicated because there is a correlation pattern that needs to be modeled and also because there are measurements that are censored. As censored observations make the traditional variance components methods not appropriate for analyzing age-at-onset data, some alternatives have been proposed. Yoo et al. [2001], for instance, proposed the use of martingale residuals from Cox survival models, adjusted without genetic random

\section{KARGER}

Fax +4161306 1234 E-Mail karger@karger.ch www.karger.com
(C) 2008 S, Karger AG, Basel

0001-5652/09/0682-0131\$26.00/0

Accessible online at:

www.karger.com/hhe
Suely Ruiz Giolo

Federal University of Parana, Department of Statistics

PO Box 19081

ZIP 81531-990 Curitiba, PR (Brazil)

Tel. +55 (11) 3069 5511, Fax +55 (11) 3069 5022, E-Mail giolo@ufpr.br 
effects, as quantitative traits in variance components models. Wintrebert et al. [2006] also proposed a methodology based on martingale residuals to estimate the presence of genetic effects as well as to estimate genetic correlations depending on the genetic distance. The methodology was applied by them for assessing genetic effects on age-at-onset of Huntington disease in a family study. However, martingale residuals from Cox models are not always normally distributed, which may invalidate the use of these methodologies. In order to analyze age-atonset data in a more appropriate manner, Pankratz et al. [2005] proposed a random effects Cox proportional hazards model, which allows for the incorporation of unmeasured random effects into the censored trait analysis, and also provides estimates for both fixed and random effects without specifying the censored trait distribution. This model was applied by authors for assessing genetic effects on age-at-onset of breast cancer in a large family study. More recently, a Bayesian shared and additive genetic random effects model for family data with longterm survivors (LTS) was proposed by Pitkäniemi et al. [2007]. In this model, a fraction of the population under study is considered to be non-susceptible or immune to the disease. Application of this model was done by authors on type 1 diabetes data. Locatelli et al. [2007] also proposed a similar Bayesian LTS model for analyzing breast cancer data in a twin study. In contrast to the model proposed by Pankratz et al. [2005], in which the baseline hazard function is left completely unspecified, the Bayesian LTS models mentioned have chosen to model the baseline hazard by Weibull and Gompertz functions, respectively.

In this paper analyses of age-at-onset of three common complex diseases associated with coronary heart disease (CHD) are performed by using data from a family based epidemiology project conducted in the Brazilian population [Oliveira et al., 2008]. Several challenges exist on the study of $\mathrm{CHD}$, since there is a complex metabolic mechanism modulating its etiology that involves both environmental and genetic components, as well as their possible interactions [Kullo and Ding, 2007]. Despite the significant heritability associated with the etiology of $\mathrm{CHD}$, it is not clear what particular phenotypes are best to be studied. Some important cardiovascular risk factors associated with $\mathrm{CHD}$ are hypertension, diabetes, and high cholesterol, which have been studied through both quantitative traits (e.g., systolic and diastolic blood pressure, fasting blood glucose, and LDL-cholesterol), and dichotomized variables (e.g., presence or not of hypertension, diabetes, and high cholesterol). Regarding the
Baependi family heart study, which we shall use in this paper, significant heritability estimates were observed for all these mentioned traits [Oliveira et al., 2008]. In order to identify genes associated with diseases, mainly in cancer and Mendelian affections, efforts have been driven to define other traits, potentially more informative, such as age-at-onset of these diseases [Pankratz et al., 2005; Wintrebert et al., 2006; Locatelli et al., 2007]. The use of these new phenotypes may not only add information to the mapping of genes associated with cardiovascular risk factors, but also contributes to the identification of broader categories of genes indirectly modulating these factors through their age-at-onset. In our analyses we evaluate genetic and environmental components involved specifically in the age-at-onset of hypertension, diabetes and high cholesterol.

In order to model the age-at-onset of the common complex diseases mentioned, we use the correlated frailty model [Pankratz et al., 2005], which allows for the estimation of unmeasured random effects such as genetic and shared family environmental components. The remainder of this paper is organized as follows. Section 2 presents details of the Baependi family heart study. Section 3 describes the model used for analyzing the data. Section 4 gives results from the analysis performed. A discussion ends the paper in Section 5.

\section{The Baependi Family Heart Study}

In this paper we analyze data from the Baependi family heart study described by Oliveira et al. [2008]. This study provides information about 119 families (1,712 individuals), living in the village of Baependi, in the state of Minas Gerais, Brazil. Data were collected between December 2005 and January 2006 in accordance with a planned sample design. Data from 631 nuclear families were available, with a size ranging from 1 to 14 offspring. The number of generations per family varied from 2 to 4 (54\% of the families had 3 generations, and $45 \%$ had 2 generations). Only individuals aged 18 years or older were considered eligible for participating in the study. The mean age was 44 years, with a range of 18 to 100 years. For each participant a questionnaire was used to obtain information regarding family relationships, demographic characteristics, medical history and environmental risk factors. Anthropometric measures, physical examination and electrocardiogram of the participants were performed by trained medical students. Also, fasting blood glucose, total cholesterol, lipoprotein fractions and 
triglycerides were obtained by standard techniques in blood samples. Serum samples were stored at $-80^{\circ} \mathrm{C}$ and genomic DNA was extracted by standard procedures [Miller et al., 1988]. Considering that families with only one or two individuals cannot provide much information where family studies are concerned, we analyzed data from 81 families, involving 1,675 people, $43.5 \%$ of whom were male. Family size varied from 3 to 157 individuals with the average being 21 per family. The average of the ages of the individuals in the pedigrees was found to be 45.5 years (s.d. $=6.58)$. Also, the minimum age among pedigrees varied from 18 to 66 years (with average of 22.85 and s.d. $=7.45$ ) and the maximum age from 46 to 100 years (the average being 74.35 and s.d. $=10.64$ ). Individuals whose glucose levels were $\geq 126 \mathrm{mg} / \mathrm{dl}$ or who were using anti-diabetic medication at the time of the study were defined as having high fasting blood glucose. Similarly, those with LDL cholesterol $\geq 160 \mathrm{mg} / \mathrm{dl}$ or using lipid lowering medication were considered having high cholesterol. Individuals with mean systolic blood pressure $\geq 140 \mathrm{~mm} \mathrm{Hg}$ and/or diastolic blood pressure $\geq 90 \mathrm{~mm} \mathrm{Hg}$ or who were taking anti-hypertensive medication at the time of the study were defined as having hypertension. Age of hypertension diagnosis for previously diagnosed individuals was defined as the age declared by them. Previously undiagnosed individuals with diastolic and/or systolic blood pressure equal to or higher than 140 and $90 \mathrm{~mm} \mathrm{Hg}$, respectively, at the time of the study, had their age at that time defined as the age of diagnosis. Individuals free of hypertension up to the end of the study had censored age of diagnosis. For diabetes and high cholesterol, age of diagnosis was defined in a similar manner. From the data of the 81 families, 596 (35.58\%) individuals were identified with hypertension, 157 (9.37\%) with diabetes and 196 (11.7\%) with high cholesterol. These results are similar to the observed prevalence of these traits in the general Brazilian population [Pereira et al., 2006].

\section{Mixed Effects Cox Model}

For censored traits such as age-at-onset or its surrogate age of diagnosis used in this paper, the traditional variance components method [Almasy and Blangero, 1998; de Andrade et al., 1999] is usually not appropriate due to asymmetry in the distribution of these traits and the presence of censored measurements. To analyze these type of traits we next outline the mixed-effects Cox model, used here for performing genetic analysis on the data of the Baependi family heart study. Let $\left(T_{i j}, \delta_{i j}, \mathbf{X}_{i j}\right)$ be the age of diagnosis, dichotomous indicator of censoring and the covariates of interest, respectively, for individual $i$ in family $j\left(i=1, \ldots, n_{j}, j=1, \ldots, k\right)$. In the mixed-effects Cox model proposed by Pankratz et al. [2005], also termed correlated frailty model, the hazard function for the $i$-th individual in family $j$ evaluated on age $t$, denoted by $\lambda_{i j}(t)$, is defined as

$$
\lambda_{i j}(t)=\lambda_{0}(t) \exp \left\{\mathbf{X}_{i j}^{\prime} \beta+g_{i j}\right\}
$$

where $\lambda_{0}(t)$ is an unspecified baseline hazard function, $\beta$ is a vector of regression coefficients for the covariates, and $g=\left(g_{11}, \ldots, g_{n_{\mathrm{k}} k}\right)$ is a vector of random effects where $g_{i j}$ is the per-subject random effect. Note that model (3.1) uses age-at-onset of the event of interest (such as hypertension or diabetes) to model the hazard (or risk) of occurrence of the disease over age $t$, where covariates and genetic effects act directly on the overall hazard function $\left(\lambda_{0}(t)\right)$ decreasing or increasing the individual hazard function $\lambda_{i j}(t)$. Assuming that the random effects of all members of all the families follow a multivariate normal distribution with mean zero and covariance matrix $\Omega$, the partial likelihood (PL) is given by

$$
L=\int \operatorname{PL}(\beta, \mathbf{g}) \frac{1}{\sqrt{2 \pi|\Omega|}} \exp \left[-\frac{1}{2} \mathbf{g}^{\prime} \mathbf{\Omega}^{-1} \mathbf{g}\right] d \mathbf{g}
$$

where PL is the Cox partial likelihood [Cox, 1975]. To evaluate simultaneously the influences of unobserved genetic and shared family environmental contributions to age-at-onset, the covariance matrix $\Omega$ can be decomposed into two components, $\Omega=2 \Phi \sigma_{g}^{2}+1 \sigma_{f}^{2}$, where $\sigma_{g}^{2}$ and $\sigma_{f}^{2}$ represent the shared polygenic effect and the shared family environmental influences, respectively. The block diagonal matrix $2 \Phi$, where each block has dimensions $n_{j} \times n_{j}$, is the so called relationship matrix that captures the shared polygenic factors between genetically related family members, and $\mathbf{1}$ is also a block diagonal matrix that incorporates the degree of shared environment among individuals of family $j$ in which each block is a matrix of dimensions $n_{j} \times n_{j}$ whose elements are all equal to 1.

As the partial likelihood (3.2) is an intractable multidimensional integral, Pankratz et al. [2005] used the Laplace approximation to the integral as defined by Ripatti and Palmgren [2000] to perform their calculations regarding this likelihood. Maximization of the resultant penalized partial likelihood over $\beta$ and $\mathbf{g}$ leads to approximate maximum likelihood estimates of the regression coefficients, as well as estimates of the variances of the random effects. This estimation approach was implemented by Pankratz's 
Table 1. Parameter estimates from Cox proportional hazards random-effects models fitted to the Baependi family data where gender, BMI and age estimates are hazard ratios, and variance components correspond to shared family $\left(\sigma_{f}^{2}\right)$ and additive polygenic $\left(\sigma_{g}^{2}\right)$ random effects

\begin{tabular}{|c|c|c|c|c|c|c|c|c|c|c|}
\hline \multicolumn{3}{|c|}{ Hypertension } & \multicolumn{3}{|l|}{ Diabetes } & \multicolumn{5}{|l|}{ Cholesterol } \\
\hline gender & $\sigma_{f}^{2}$ & $\sigma_{g}^{2}$ & BMI & $\sigma_{f}^{2}$ & $\sigma_{g}^{2}$ & gender & age & BMI & $\sigma_{f}^{2}$ & $\sigma_{g}^{2}$ \\
\hline \multicolumn{11}{|c|}{ Model: No random effect } \\
\hline $\begin{array}{l}1.53 \\
(1.29-1.80)\end{array}$ & & & $\begin{array}{l}1.08 \\
(1.04-1.11)\end{array}$ & & & $\begin{array}{l}1.46 \\
(1.07-1.98)\end{array}$ & $\begin{array}{l}0.81 \\
(0.78-0.84)\end{array}$ & $\begin{array}{l}1.01 \\
(0.99-1.06)\end{array}$ & & \\
\hline \multicolumn{11}{|c|}{ Model: Shared family } \\
\hline $\begin{array}{l}1.56 \\
(1.31-1.84)\end{array}$ & $\begin{array}{l}0.09 \\
(0.03-0.20)\end{array}$ & & $\begin{array}{l}1.07 \\
(1.03-1.10)\end{array}$ & $\begin{array}{l}0.27 \\
(0.06-0.65)\end{array}$ & & $\begin{array}{l}1.47 \\
(1.08-2.00)\end{array}$ & $\begin{array}{l}0.81 \\
(0.78-0.84)\end{array}$ & $\begin{array}{l}1.01 \\
(0.99-1.05)\end{array}$ & $\begin{array}{l}0.15 \\
(0.02-0.42)\end{array}$ & \\
\hline \multicolumn{11}{|c|}{ Model: Polygenic } \\
\hline $\begin{array}{l}1.67 \\
(1.35-2.05)\end{array}$ & & $\begin{array}{l}0.79 \\
(0.44-1.29)\end{array}$ & $\begin{array}{l}1.08 \\
(1.04-1.12)\end{array}$ & & $\begin{array}{l}0.91 \\
(0.28-1.92)\end{array}$ & $\begin{array}{l}1.47 \\
(1.07-2.01)\end{array}$ & $\begin{array}{l}0.81 \\
(0.78-0.84)\end{array}$ & $\begin{array}{l}1.01 \\
(0.99-1.05)\end{array}$ & & $\begin{array}{l}0.36 \\
(0.0-0.88)\end{array}$ \\
\hline \multicolumn{11}{|c|}{ Model: Polygenic and shared family } \\
\hline $\begin{array}{l}1.67 \\
(1.36-2.05)\end{array}$ & $\begin{array}{l}0.05 \\
(0.0-0.18)\end{array}$ & $\begin{array}{l}0.69 \\
(0.36-1.19)\end{array}$ & $\begin{array}{l}1.07 \\
(1.04-1.11)\end{array}$ & $\begin{array}{l}0.20 \\
(0.0-0.63)\end{array}$ & $\begin{array}{l}0.59 \\
(0.0-1.55)\end{array}$ & $\begin{array}{l}1.47 \\
(1.07-2.01)\end{array}$ & $\begin{array}{l}0.81 \\
(0.78-0.84)\end{array}$ & $\begin{array}{l}1.01 \\
(0.99-1.05)\end{array}$ & $\begin{array}{l}0.12 \\
(0.0-0.38)\end{array}$ & $\begin{array}{l}0.14 \\
(0.0-0.67)\end{array}$ \\
\hline
\end{tabular}

group in the coxme function of the $\mathrm{R}$ kinship library $[\mathrm{R}$ Development Core Team, 2008]. Because it is very difficult to obtain the standard errors (SE) for the variance components, confidence intervals are obtained by profiling the likelihood. Another way to calculate the SE is to perform bootstrap [de Andrade et al., 2006].

A particularity regarding the mixed-effects Cox model (3.1) is that for such model it is not possible to obtain direct heritability estimates as in the variance components model, since there is no random error variance component. However, the variance components obtained from model (3.1) may be interpreted as measures of familial aggregation. Information concerning the relative risk of the disease that corresponds to the random effect is obtained by exponentiation of the square root of the variance component. Relative risks associated with the covariates can also be obtained by exponentiation of each regression coefficient. In this paper, the criterion established for selecting the covariates to be maintained in model (3.1) was to choose those covariates leading to the highest estimates of the polygenic variance component.

\section{Results}

We fitted a series of mixed-effects proportional hazards models for analyzing the Baependi family data in order to asses the influence of genetic and shared family environmental factors in age-at-onset of the cardiovascular risk factors: hypertension, diabetes and high choles- terol. Since age-at-onset is difficult to access, we have used age of diagnosis as its surrogate. The first model fitted corresponds to the standard proportional hazards model which ignores any potential statistical dependencies between individuals due to common genes or environment. The second model corresponds to the shared family model in which all members of a family are assumed to share the same unmeasured risk. This model incorporates a single random effect per family which varies across families with variance given by the estimated variance component $\sigma_{f}^{2}$. The third model corresponds to the shared polygenic model which incorporates one random effect per subject that are correlated across individuals within the family according to the degree of their relationships. An estimate of the polygenic variance component, $\sigma_{g}^{2}$, is provided by this model. The last model simultaneously includes both shared polygenic and shared family environmental effects with variance components $\sigma_{g}^{2}$ and $\sigma_{f}^{2}$ provided. The covariance matrices associated with the three last models are $\Omega=1 \sigma_{f}^{2}, \Omega=$ $2 \Phi \sigma_{g}^{2}$, and $\Omega=2 \Phi \sigma_{g}^{2}+1 \sigma_{f}^{2}$, respectively. For all fitted models, we present in table 1 a summary of the parameter estimates and their 95\% confidence intervals where the columns labeled gender, age, and BMI (body mass index) are risk ratios estimates (i.e, $\exp (\hat{\beta}))$, and $\sigma_{f}^{2}$ and $\sigma_{g}^{2}$ correspond to shared family and additive polygenic variance components, respectively. Although models with different covariates have been fitted, in table 1 we present the results only for the group of covariates that produced the highest estimates of the polygenic variance components 
in accordance with the selection criterion mentioned in Section 3.

The final model for age-at-onset of hypertension included only the covariate gender, coded as 0 for male and 1 for female. Although age and BMI are covariates usually considered in the risk evaluation of hypertension, in our model their effects were absorbed by the polygenic variance component. Thus, age and BMI were not kept in our final model. Under the standard proportional hazards model the estimated risk of hypertension among females was approximately $53 \%$ greater than the risk among males. A moderate increase in this estimate can be observed in the other three fitted models. Opposite than usually expected, note that women of the Baependi study presented higher risks for hypertension than men. This result might probably be a characteristic of this part of the Brazilian population. Lotufo et al. [1998], for instance, showed that Brazilian cities had a high mortality pattern of heart diseases, compared with other countries, mainly among women. Additionally, Firmo et al. [2004] assessing factors associated with awareness of hypertension among older adults living in a community similar of Baependi, showed an association of female gender with awareness of being hypertensive. More recently, Bassanezi et al. [2006] also reported that premature mortality due to cardiovascular diseases among women is higher in Brazil than in other countries and showed that almost half of this mortality can be attributed to social inequalities.

From the shared family model fitted for age-at-onset of hypertension, which incorporates one random effect per family, the estimated variance component for shared family environmental effect was 0.09 , indicating that the average of relative risk among families is $\exp (\sqrt{0.09})=$ 1.35 , with $95 \%$ confidence interval (CI) of 1.19-1.56. Hence, all members of a particular family may have risk of hypertension which is on average $35 \%$ higher than the overall average risk for the entire sample, reflecting a moderate degree of familial aggregation for hypertension. This model, however, assigns the same relative risk for all members of a family without taking into account genetic relationships. From the polygenic model which incorporates one random effect per subject, where such random effects are correlated according to the degree of their relationships, we obtained an estimate of the polygenic variance component equal to 0.79 , with a $95 \%$ confidence interval of $0.44-1.29$. This result suggests a significant degree of heritability associated to the age-at-onset of hypertension and shows that the individual relative risks of hypertension due to polygenic effects are on average $\exp (\sqrt{0.79})=2.4$. Then, there are individuals which may have risk of hypertension $140 \%$ higher than the overall average risk for the entire sample. In order to evaluate the contributions of each of these two components of unmeasured risk, we consider both polygenic and shared family environmental random effects simultaneously in the model. Under this model, the estimated polygenic and shared family variance components were 0.69 (95\% CI, 0.36-1.19) and 0.05 (95\% CI, 0.0-0.18), respectively. From these results, there is evidence that polygenic factors play a greater role than shared family environment factors in explaining the variation in the age-at-onset of hypertension. The relative risk of hypertension corresponding to an additive genetic component varies from 82 to $197 \%$ of the overall average in the Baependi family heart study. From all of these results we can notice that family history might significantly modify the estimates of individual risk of hypertension. Figure 1 displays estimates of the additive polygenic relative risks for all members of family 48 in the pedigree plot. Information concerning the age and BMI of all individuals, as well as the age of diagnosis of those individuals having hypertension, are also presented in the pedigree plot. For this family the shared family model assigns the same familial risk of 1.3 to all members. From members with diagnosis of hypertension in this family we can see that age of diagnosis is in general younger for females. Also, the male member aged 25 years whose mother and grandmother had diagnosis of hypertension at 27 and 19 years old and BMI of 38 and 35, respectively, has a relative risk of hypertension greater than, for instance, that one aged 31 years but whose mother had diagnosis of hypertension later. As previously mentioned, this result illustrates that age and BMI effects have been absorbed by the polygenic variance component on the risk evaluation.

Concerning the cardiovascular risk factor diabetes, the final model included the covariate body mass index (BMI). The gender effect was not significant in the presence of BMI, and the age effect was absorbed by the polygenic variance component. For the BMI covariate, the estimated relative risk of diabetes was 1.08 (95\% CI, 1.041.11). This estimate was practically unchangeable for all models. Thus, as BMI increases, the risk of diabetes also rises. From the results of the shared family model (table 1), we found a per-family risk of diabetes of $\exp (\sqrt{0.27})=1.68$. It indicates that all members of a particular family may have risk $68 \%$ higher than the overall risk of diabetes for the entire sample, reflecting a degree of familial aggregation of diabetes more substantial than that one observed for hypertension. A significant degree of heritability to the age-at-onset of diabetes is also sug- 
Fig. 1. Pedigree plot for family 48 where age, BMI and age of diagnosis (for those having hypertension - solid individuals) are printed together with the polygenic relative risk estimates (between parentheses). Common relative risk shared among all family members is 1.3 .

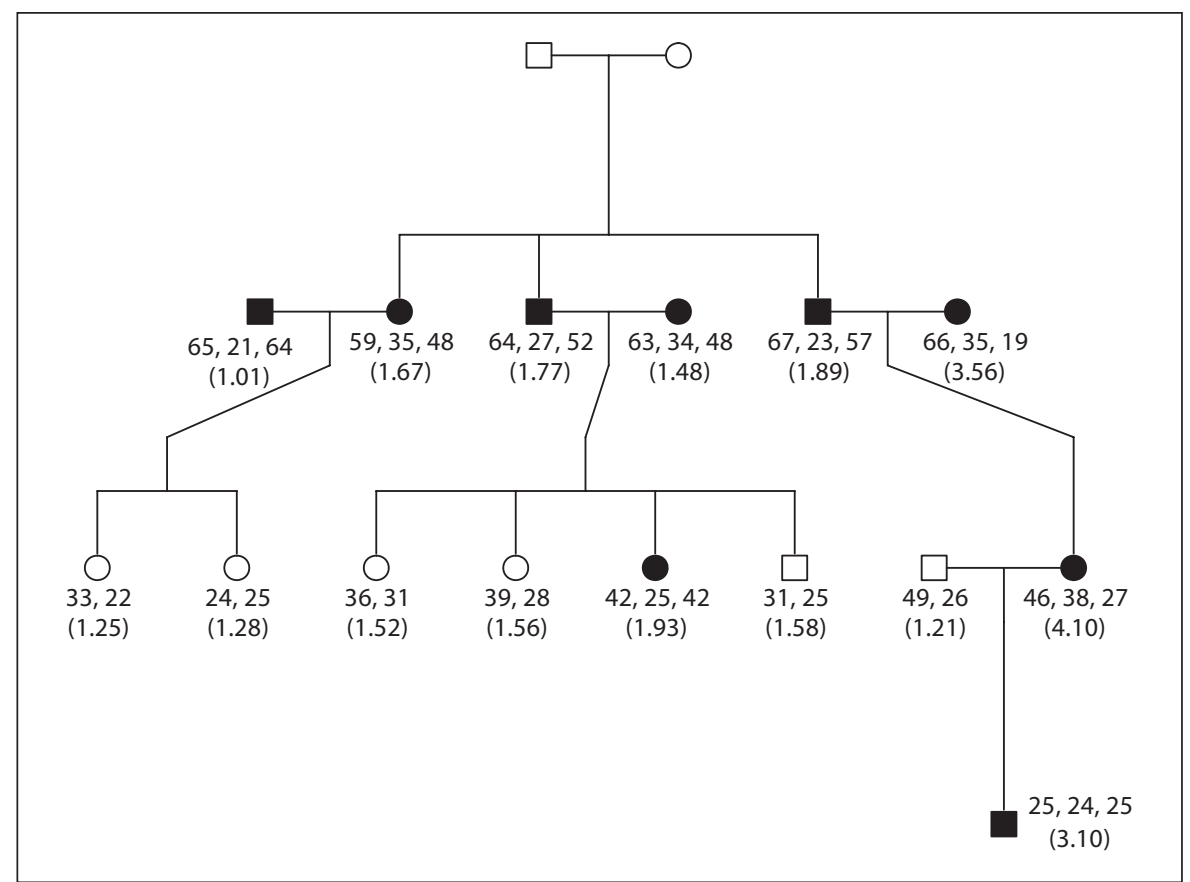

gested from the estimate of the polygenic covariance component. From that estimate ( 0.91 with a $95 \%$ CI of $0.28-1.92)$ the relative risk of diabetes due to polygenic influences is $\exp (\sqrt{0.91})=2.6$ indicating that there are individuals which may have risk $160 \%$ higher than the overall average risk for the entire sample. The model that simultaneously includes both random effects (polygenic and shared family) suggests from the estimates of the polygenic and shared family variance components, 0.59 and 0.20 , respectively, that both factors might play a role in explaining the variation in the age-at-onset of diabetes, with the polygenic effect playing a greater role.

From the analyses performed with regard to age-atonset of high cholesterol, the final model included the covariates gender, age and BMI. In this case, age and BMI effects were found not to be associated with the polygenic effect given that their inclusions have not changed the polygenic variance component estimates. For the covariates gender, age and BMI remaining in the model the estimated relative risks of high cholesterol were $1.46(95 \%$ CI, 1.07-1.98), 0.81 (95\% CI, 0.78-0.84), and 1.01 (95\% CI, 0.98-1.05), respectively. Thus, females have a higher risk of high cholesterol. Also, the risk of high cholesterol decreases with age. Conversely, this risk increases with BMI. From the shared family model, all member of a particular family may have a risk of high cholesterol $47 \%$ (95\% CI, 10-92\%) higher than the overall average risk, suggesting a moderate degree of familial aggregation of high cholesterol. The estimate of 0.36 for the polygenic variance component in the polygenic model was smaller than those obtained in the analyses of age-at-onset of hypertension and diabetes. From the most general model (polygenic and shared family) similar estimates of the polygenic and shared family covariance components were obtained, 0.12 and 0.14 , respectively.

\section{Discussion}

In this paper we analyzed the data of 1,675 individuals from 81 Brazilian families to evaluate the effect of genetic and environmental factors on age-at-onset of three cardiovascular risk factors: hypertension, diabetes and high cholesterol. Based on our results analyzing the ages of onset of these traits with a mixed-effects Cox model, we found evidence of significant polygenic effects showing that age-at-onset is a useful trait for gene mapping of these three common complex diseases. From the models that simultaneously examined shared family and polygenic random effects, the polygenic contribution was found to play a greater role in explaining the variability of the age-at-onset of these diseases. Other authors looking at genetic and common environment heritabilities of some traits such as blood pressures, lipids and body mass 
index, among others, have also found similar small environmental effects and much larger genetic effects [Hunt et al., 1989; McCaffery et al., 1999; Saunders and Gulliford, 2006]. In our analyses, polygenic contribution was also found to be higher for hypertension than diabetes and high cholesterol. Additionally, a degree of familial aggregation was only observed in the explanation of the inter-individual variability of the age-at-onset of diabetes. Analyzing the Baependi data, evidence of a significant polygenic effect was also found by Oliveira et al. [2008] concerning the quantitative and dichotomized traits commonly used for analyzing these diseases (systolic and diastolic blood pressure, fasting blood glucose, total cholesterol, among others). From our analyses performed on ages of diagnosis of these diseases we found evidence that genetic factors can also explain a proportion of the variability of the age of the diagnosis (or ageat-onset) of these diseases. Since Oliveira et al. [2008] found evidence of significant polygenic effects associated with the dichotomized trait diagnosis of the disease as we found for age of diagnosis of the disease, genome wide mapping could confirm whether there is a common set of genes involved in each of these traits. However, both analyses are essentially different and their results cannot be directly compared. The model for dichotomized traits (affected/unaffected) is based on the liability threshold model [Lynch and Walsh, 1998] which assumes a risk function for phenotypes in a continuous unobserved (underlying) scale where age-at-onset is not necessarily incorporated in that function. Additionally, analysis of dichotomized traits has some undesirable features such as the subjectivity of the choice of the threshold that can produce discrepant results, and also that there is no unbiased conversion between observed and unobserved (underlying) scale heritabilities [Duggirala et al., 1997; Lynch and Walsh, 1998]. The Cox model used in the current paper models the disease risk as a function of the age-at-onset allowing for censored observations and asymmetry in the distribution of the age-at-onset. The Cox model can also provide some additional information not given by the analysis of dichotomized traits. For instance, we can estimate the risk of hypertension of a particular individual (male or female) at any age, but it cannot be obtained under the liability threshold model.

Concerning the risk evaluation of cardiovascular diseases, gender, age and BMI are usually considered in the analysis as covariates, but little has been done in terms of the familial history contribution to the risk estimates. In this paper we dealt with this subject by showing that the polygenic random component might absorb the effect of some of these covariates, increasing or decreasing the individual relative risks. The hypertension risk evaluation, for instance, showed that gender is a significant factor, and also that age and BMI are associated with the polygenic component. For diabetes, the gender effect was not significant in the presence of BMI, and the age effect was absorbed by the polygenic variance component. Finally, for high cholesterol, gender, age and BMI were significant, with the last two being not associated with the polygenic effect, given that their inclusions in the model have not changed the polygenic variance component estimates.

Although for genetic studies there are many parallels of the mixed-effects Cox model with classical variance components methods [Pankratz et al., 2005], it is not possible under the Cox model to obtain direct heritability estimates. However, relative risks can be computed from the variance components allowing us to make similar statements as those provided by heritability estimates. As an effort to obtain heritability estimates for age-at-onset of the diseases analyzed in this paper, we performed the analysis (not shown) proposed by Yoo et al. [2001], which uses the martingale residuals from Cox models. However, the required normality assumption was not verified, not allowing that estimation. In animal breeding, heritability estimates have been investigated under parametric (Weibull) survival models by Yazdi et al. [2002]. Even though heritability is an informative measure for quantitative genetic studies, cautions need to be taken in terms of its interpretation, because it might reflect a proportion of an overall variance component of very low magnitude. Similar cautions are true concerning the relative risks obtained from Cox models. Under the Cox model, however, a benefit that can be exploited is the possibility to visualize the pedigree information combined to the individual relative risks estimates due to the random and fixed effects, as illustrated in figure 1. Such information might help in clinical advisements.

An important consideration in the analysis of occurrence of a condition is the subjectivity of the definition of the condition by dichotomizing a continuous measurement. Problems caused by this subjectivity may be exacerbated considering that a high percentage of people is expected with these common diseases not diagnosed due to the deficiency in the Brazilian Health Care System [Fleury et al., 2000]. Longitudinal family studies might probably reduce the effect of these false negative diagnoses. For quantitative traits, genetic analysis of longitudinal family data has been performed by multivariate approaches [Amos et al., 2001]. Although in this paper the mixed-effects Cox model was used to analyze a cross-sec- 
tional family data, extensions of it to accommodate longitudinal family data and also time-dependent covariates are possible [Martinussen and Scheike, 2006]. The application of the Cox model for analyzing rare and common diseases can also be extended to accommodate situations in which there are forces accelerating or decelerating the time to event of interest. The Birnbaum-Saunders [Birnbaum and Saunders, 1969; Kundu et al., 2008] parametric LTS models [Locatelli et al., 2007, Pitkäniemi et al., 2007] and also a nonparametric LTS model, for instance, might be appropriate alternatives for modeling each one of these situations. Since genetic and environmental factors might be involved in that acceleration or deceleration process, extending these models in genetic analysis would be useful.

\section{Acknowledgement}

The authors thank the reviewers and the associate editor for their constructive comments and suggestions as well as the $\mathrm{CNPq}$ (Brazil, Grant 150653/2008-5) for partial financial support to the first author.

\section{References}

Almasy L, Blangero J: Multipoint quantitativetrait linkage analysis in general pedigrees. Am J Hum Genet 1998;62:1198-1211.

-Amos C, de Andrade M, Zhu D: Comparison of multivariate tests for genetic linkage. Hum Hered 2001;51:133-144.

-Bassanesi SL, Azambuja MI, Achutti A: A Premature mortality due to cardiovascular disease and social inequalities in Porto Alegre: from evidence to action. Arq Bras Cardiol 2008;90:403-412.

-Birnbaum ZW, Saunders SC: A new family of life distributions. J Appl Prob 1969;6:319-327.

Cox DR: Partial likelihood. Biometrika 1975;62: 269-276.

de Andrade M, Amos CI, Thiel TJ: Methods to estimate genetic components of variance for quantitative traits in family studies. Genet Epidemiol 1999;17:64-76.

de Andrade M, Atkinson EJ, Lunde E, Amos CI, Chen J: Estimating genetic components of variance for quantitative traits in family studies using the MULTIC routines. Copywrite Mayo Foundation: Technical Report \#78, 2006. http://mayoresearch.mayo.edu/ mayo/research/biostat/techreports.cfm

- Duggirala R, Williams JT, Williams-Blanglero S, Blanglero J: A variance component approach trait linkage analysis using a threshold model. Genet Epidemiol 1997;14:987-992.

-Hunt SC, Hasstedt SJ, Kuida H, Stults BM, Hopkins PN, Williams RR: Genetic heritability and common environmental components of resting and stressed blood pressures, lipids, and body mass index in Utah pedigrees and
twins. Am J Epidemiol 1989;129:625-638.

Lynch M, Walsh JB: Genetics and analysis of quantitative traits. Sinauer, Sunderland, 1998, pp 980.

-McCaffery JM, Pogue-Geile MF, Debski TT, Manuck SB: Genetic and environmental causes of covariation among blood pressure, body mass and serum lipids during young adulthood: a twin study. J Hypertens 1999; 17:1677-1685.
Firmo JO, Ucha E, Lima-Costa MF: The Bambui Health and Aging Study (BHAS): factors associated with awareness of hypertension among older adults. Rep Public Health 2004; 20:512-521.

Fleury S, Belmartino S, Baris E: Reshaping Health Care in Latin America: a Comparative Analysis of Health Care Reform in Argentina, Brazil, and Mexico. 2000. IDCR Books online, assessed on 08 Aug 2008 (http://www.idrc.ca/en/ev-9421-201-1-DO. TOPIC.html).

Kullo IJ, Ding K: Mechanisms of disease: the genetic basis of coronary heart disease. Nat Clin Pract Cardiovasc Med 2007;4:558-569.

Kundu D, Kannan N, Balakrishnan N: On the hazard function of Birnbaum-Saunders distribution and associated inference. Comput Stat Data Anal 2008;52:2692-2702.

Locatelli I, Rosina A, Lichtenstein P, Yashin AI: A correlated frailty model with long-term survivors for estimating the heritability of breast cancer. Stat Med 2007;26:3722-3734.

Lotufo PA: Premature mortality from heart diseases in Brazil. A comparison with other countries. Arq Bras Cardiol 1988;70:321325.

Martinussen T, Scheike TH: Dynamic regression models for survival data. New York, Springer Verlag, 2006.

Miller SA, Dykes DD, Polesky HF: A simple salting out procedure for extracting DNA from human nucleated cells. Nucleic Acids Res 1988;16:1215.

- Oliveira CM, Pereira AC, de Andrade M, Soler JMP, Krieger JE: Heritability of cardiovascular risk factors in a Brazilian population: Baependi Heart Study. BMC Med Genet 2008;9:32.

Pankratz VS, de Andrade M, Therneau T: Random-effects cox proportional hazards model: general variance components methods for time-to-event data. Genet Epidemiol 2005; 28:97-109.
Pereira AC, Sposito AC, Mota GF, Cunha RS, Herkenhoff FL, Mill JG, Krieger JE: Endothelial nitric oxide synthase gene variant modulates the relationship between serum cholesterol levels and blood pressure in the general population: new evidence for a direct effect of lipids in arterial blood pressure. Atherosclerosis 2006;184:193-200.

Pitkäniemi J, Moltchanova E, Haapala L, Harjutsalo V, Tuomilehto J, Hakulinen T: Genetic random effects model for family data with long-term survivors: analysis of diabetic nephropathy in type 1 diabetes. Genet Epidemiol 2007;31:697-708.

R Development Core Team R: A Language and Environment for Statistical Computing. $\mathrm{R}$ Foundation for Statistical Computing, Vienna, Austria, 2008. ISBN 3-900051-07-0, URL http://www.R-project.org.

Ripatti S, Palmgren J: Estimation of multivariate frailty models using penalized partial likelihood. Biometrics 2000;56:1016-1022.

-Saunders CL, Gulliford MC: Heritabilities and shared environmental effects were estimated from household clustering in national health survey data. J Clin Epidemiol 2006;59:11911198.

Wintrebert CMA, Zwinderman AH, Maat-Kievit A, Roos RA, van Houwelingen HC: Assessing genetic effects in survival data by correlation martigale residuals with ana application to age at onset of Huntington disease. Stat Med 2006;25:3190-3200.

-Yazdi MH, Visscher PM, Ducrocq V, Thompson R: Heritability, reliability of genetic evaluations and response to selection in proportional hazards models. J Dairy Sci 2002;85: 1563-1577.

-Yoo B, Pankratz S, de Andrade M: Practical application of residuals from survival models in quantitative trait linkage analysis. Genet Epidemiol 2001;21(suppl 1):S811-S816. 